\title{
Vertebral Shape: Automatic Measurement with Dynamically Sequenced Active Appearance Models
}

\author{
M.G. Roberts, T.F. Cootes, and J.E. Adams \\ Department of Imaging Science and Biomedical Engineering, \\ University of Manchester, \\ Manchester, M13 9PL, UK \\ martin.roberts@manchester.ac.uk
}

\begin{abstract}
The shape and appearance of vertebrae on lateral dual x-ray absorptiometry (DXA) scans were statistically modelled. The spine was modelled by a sequence of overlapping triplets of vertebrae, using Active Appearance Models (AAMs). To automate vertebral morphometry, the sequence of trained models was matched to previously unseen scans. The dataset includes a significant number of pathologies. A new dynamic ordering algorithm was assessed for the model fitting sequence, using the best quality of fit achieved by multiple sub-model candidates. The accuracy of the search was improved by dynamically imposing the best quality candidate first. The results confirm the feasibility of substantially automating vertebral morphometry measurements even with fractures or noisy images.
\end{abstract}

\section{Introduction}

\subsection{Context - Osteoporosis and Vertebral Fracture}

Osteoporosis is a progressive skeletal disease characterized by a reduction in bone mass, resulting in an increased risk of fractures. Vertebral fractures are the most common, and their presence significantly increases the risk of further vertebral and non-vertebral fractures [7. The accurate identification of prevalent vertebral fractures is therefore clinically important. However there is no precise definition of exactly what constitutes a vertebral fracture, though a variety of methods of describing them have been developed [1. These include semi-quantitative methods involving some subjective judgement by an expert radiologist, and fully quantitative morphometric methods. The latter require the manual annotation of six (or more) points on each vertebra. This annotation is time consuming, and subtle shape information is lost in the reduction of shape to 6 points.

Our ultimate aim is to define more reliable quantitative fracture classification methods based on a complete definition of the vertebra's shape. The first step must therefore be to achieve a reliable automatic segmentation. Some success in automatically locating vertebrae has been reported by several authors 8963. The purpose of this study was firstly to assess the feasibility of using an Active 
Appearance Model (AAM) [11] approach to a challenging dataset, including fractured vertebrae, and noisy images. A second aim was to improve the accuracy and robustness of the techniques by improving the sequence in which vertebral locations are determined.

\section{Materials and Methods}

\subsection{Data - DXA Images}

Assessment for vertebral fracture is traditionally carried out using spinal radiographs. This study used dual x-ray absorptiometry (DXA) images however. Despite the images being noisier and of lower resolution, DXA has several advantages, such as a substantially lower radiation dose, and a lack of projective effects. Figure 1a shows a typical DXA scan with some endplate fractures, and Figure 1b shows the solution superimposed. The models cover the lumbar starting at L4 and continuing up the thorax up to vertebra T7.

The DXA images used were obtained from two previous studies [8] ], obtained from a Hologic (Bedford, MA) QDR2000plus scanner and a QDR4500A scanner respectively. Pixel dimensions are $1.0 \mathrm{~mm}$ and $0.5 \mathrm{~mm}$ respectively. The combined dataset of 202 images contains 173 fractures in total, and many images are very noisy due to an inherent bias towards obese patients in the clodronate study (second dataset). The images were manually annotated using an in-house tool, by one of the authors (MGR), with supervision by an experienced radiologist
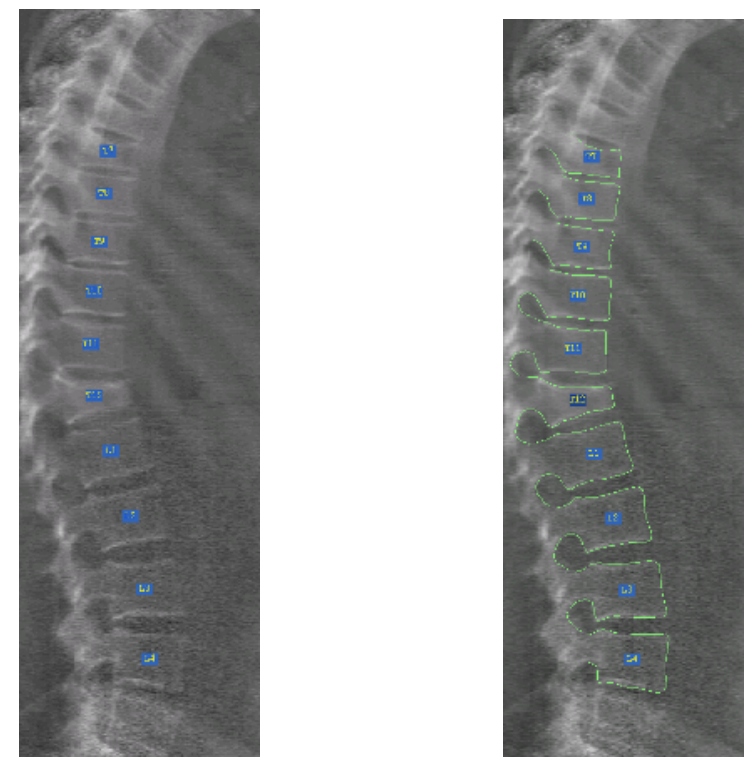

Fig. 1. Lateral DXA image of a spine displaying some symptoms of osteoporosis (e.g. T12 fracture). a) shows the raw image; b) shows the model solution superimposed. 
(JEA). Each vertebral contour uses between 32 to 40 points around the vertebral body (32 points for T10 and above), with 8 further points around the pedicles for L4 to T10.

\subsection{Statistical Models in Medical Imaging}

Many problems in medical image interpretation require an automated system to interpret images. These images may provide noisy data, and typically complex structure. Model based methods offer solutions to these difficulties, by enforcing strong priors learned from a set of annotated training images. A widespread such current approach is the Active Appearance Model (AAM) [11.

One fundamental problem is that under-training of the model means that it may be insufficiently adaptable on a local level, especially when pathologies are present. In previous work 9] we showed that this problem could at least be mitigated by using multiple sub-models. The sub-structures were linked by partially overlapping them, and using the constrained form of the AAM [10].

\subsection{Combining Multiple Sub-models}

In $[9$ we demonstrated this approach on Smyth's original dataset $[8$ of predominantly normal spines. We modelled the spine by using a sequence of overlapping triplets of vertebrae. In this paper we improve the robustness and generality of the algorithm by developing a dynamic sequencing method for the order in which the sub-models are fit.

The sequence of sub-model solutions were combined in 9] as follows, using a fixed ordering starting by going up the lumbar. Each vertebra is fit using the triplet sub-model in which it is central (Figure 2). Note that subsequent iterations do not update any point positions which have already been determined, unless the point is in the central vertebra of the triplet. When a triplet model has been fit it provides part of the initialisation (via the overlapping vertebrae) for subsequent iterations which fit its neighbours. Furthermore constraints are applied [10] so that overlapping vertebrae cannot be moved far from the provisional positions determined previously. This feed-forward of constraints is further aided by re-fitting the global shape model to the solution so far. This is used to initialise a starting solution for vertebrae not yet fit, and relatively low constraint weights are attached to this global prior. Thus information in the global shape model is still used in guiding the solution, but the global shape constraints are downweighted, which allows their violation to a degree if the image evidence locally supports such a solution.

Our approach differs somewhat from that of Davatzikos et al 2], who propose a hierarchical Active Shape Model based on a wavelet decomposition of the shape contours, in which local regions of the wavelet transform space are decoupled when applying the shape model constraints. In [2] coarse global constraints continue to apply in a strict sense. However, in the case of vertebral morphometry, certain pathologies of the spine such as scoliosis may cause even coarse aspects of the shape model to be violated, as whole vertebrae can be laterally 

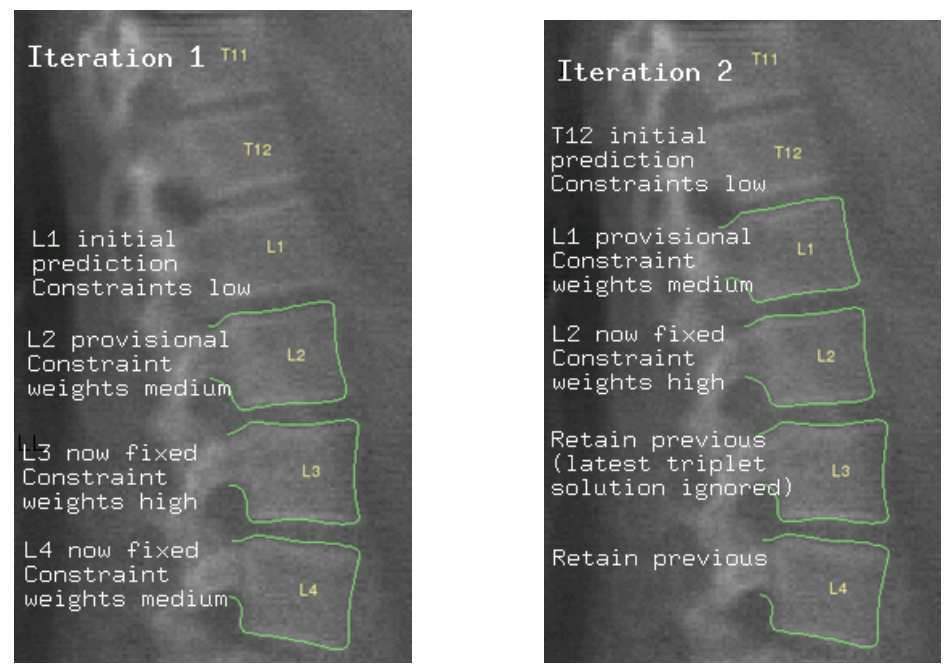

Fig. 2. An illustration of the first two iterations (static fit ordering) combining vertebral triplet sub-models. a) shows the result of fitting the first triplet containing L4/L3/L2. b) indicates the next iteration which fits L3/L2/L1. The second iteration resets $\mathrm{L} 2$ and provides an initialisation of L1, and an updated prediction for T12 via the global shape model.

shifted outside the region captured in the training set. Therefore we use multiple overlapping AAMs instead, which allows a wider range of pathologies to be adequately fitted. Furthermore there may be some advantages in decomposing the image search process, as well as the application of shape constraints.

\subsection{Dynamic Sub-model Sequence Ordering Algorithm}

If one sub-model fit fails, then this can misalign the starting solution for subsequent iterations. In such a case it would be better to adapt the sequence dynamically by comparing the fit quality of several candidate sub-models. Picking the best fitting model as the one to impose at this iteration will tend to defer noisier or poorly fitting regions until they have been better constrained by their neighbours.

In this new approach a subset of $N$ active candidate sub-models is maintained. Each such candidate is provisionally fitted, then the submodel with the best quality of fit (see next section) is imposed into the overall solution, and removed from the candidate list. A new candidate sub-model to replace the one just imposed is added at the end of each iteration by searching from the latest best candidate to locate its nearest remaining neighboun. As with the static ordering, the global shape model is then fitted to the subset of all points determined so far, and used to predict an initial solution for all points which have not yet formed part of any imposed sub-model solution. These iterations continue

1 "Remaining" means that the submodel has never been in the candidate list. 
until all sub-models have either been fitted and imposed already, or are now in the set of candidates. When no more candidates can be added the remaining $N-1$ sub-models are fitted (best quality first) and the search concludes. It is to be noted that all candidates in the active subset are re-fitted at each iteration, as they will generally have a slightly different initialisation and altered shape constraints as a consequence of imposing the last accepted candidate. We used a set of 3 candidate sub-models, starting with the top, bottom and middle of the spine.

\subsection{Quality of Fit Measure}

The quality of fit measure used to pick the best candidate sub-model is based on the scaled residual sum of squares $S$, which is calculated as:

$$
S=\sum_{i=1}^{n} \frac{r_{i}^{2}}{\hat{\sigma}_{i}^{2}}
$$

where $r_{i}$ is the grey level residual at point $i$, and $\hat{\sigma}_{i}$ is its estimated standard deviation. However, when comparing different sub-models with different numbers of points it is not meaningful to directly compare values of $S$. Instead we pick the best fitting sub-model, in the sense of having the lowest probability of obtaining a residual sum of squares any better (i.e. lower) than that achieved. So $S$ is mapped to a value $z$ on a standard Gaussian approximating the theoretically $\chi^{2}$ distribution of $S$, but with further scaling parameters $\alpha$ and $\beta$ for its overall mean and variance. These scaling parameters are necessary because the set $\hat{\sigma}_{i}$ are underestimated, as they are calculated at model creation by refitting the model to the training set, and do not account for model inadequacies in fitting to unseen images. To more accurately model the true distribution of $S$, the boosting parameters $\alpha$ and $\beta$ in mean and variance due to model inadequacy are derived by running a preliminary set of randomised train/test set partitions. The final standardised Gaussian z-value used is then:

$$
z=\frac{S-\alpha n}{\alpha \sqrt{2 n \beta}}
$$

The quality measure is obtained by just negating this, as picking the highest value of $-z$ is equivalent to choosing the candidate with the lowest probability of obtaining a residual sum of squares any better than that achieved.

\subsection{Experiments}

Leave-8-out tests were performed over the 202 images. When the algorithm is run interactively in an associated prototype clinical tool, the clinician initialises the solution by clicking on 3 points. These are the bottom of L4, top of T12 and top of T7. The global shape model fit to these 3 points is used as the starting solution. On each experiment the 3-point initialisation was simulated by using the known equivalent marked points and adding random offsets to 
them. These were zero-mean Gaussian errors with SD of $1 \mathrm{~mm}$ in the y-direction (along the spine) and $3 \mathrm{~mm}$ in the $\mathrm{x}$-direction. Twenty replications (i.e. random initialisations) of each image were performed. The AAMs sampled the gradient along profiles spanning $6 \mathrm{~mm}$ either side of the shape, with the overall sample renormalised onto the sample cdf.

\section{Results}

The accuracy of the search was characterised by calculating the absolute pointto-line distance error for each point on the vertebral body. The error is the distance from each located point to the nearest point (in the same vertebra) on the smooth bezier spline passing through the manually annotated points.

\subsection{Optimal Sub-models}

We assessed the effect of the size of structures used for the sub-models. As well as triplets, we tried using individual vertebra models, and a central vertebra with the neighbouring half-vertebrae. Scaling up the structure size, we assessed the use of quintets ( 5 vertebrae). Space does not permit the inclusion of full results. In summary, structures smaller than triplets were found to be too unconstrained and therefore unreliable. Triplets performed slightly better than quintets on fractured vertebrae, but there was little difference between triplets and quintets on normal vertebrae. We concluded that triplets are the optimal sub-structure to model.

\subsection{Dynamic vs Static Ordering}

The overall mean point accuracies are $0.79,1.03$, and $0.92 \mathrm{~mm}$ for the dynamically ordered, statically ordered, and global model approaches respectively. Table 1 compares results for static and dynamic sub-model ordering, but decomposing the data into points within normal or fractured vertebrae. Each row gives the mean, median and 75 th percentiles, and the percentage of point errors in excess of $2 \mathrm{~mm}$. The threshold of $2 \mathrm{~mm}$ would be around 2SDs of manual precision, and can be viewed as a fairly stringent point failure indicator. The Eastell morphometric method [5] was used to determine fracture status.

Comparing the dynamic and static ordering results in table 1 , there is an improvement by using the new dynamic ordering method, but this is mainly

Table 1. Search Accuracy Percentiles by Fracture Status

\begin{tabular}{|l|cccl|cccc|}
\hline \multicolumn{5}{|c|}{ Normal } & \multicolumn{4}{c|}{ Fractured } \\
\hline Model Fit & Mean & Median & $75 \%$ ile & ge errors & Mean Median & $75 \%$ ile \%ge errors \\
Strategy & Acc & Acc & Acc & over 2mm & Acc & Acc & Acc & over 2mm \\
\hline Dynamic & 0.70 & 0.50 & 0.92 & $4.96 \%$ & 1.23 & 0.70 & 1.41 & $16.22 \%$ \\
Static & 0.88 & 0.55 & 1.04 & $7.54 \%$ & 1.80 & 0.83 & 1.79 & $22.21 \%$ \\
Global & 0.84 & 0.62 & 1.12 & $7.12 \%$ & 1.37 & 0.84 & 1.63 & $18.17 \%$ \\
\hline
\end{tabular}


evident in the tails of the error distribution. Although there is little difference in median accuracy, the reduction in extent of the error tails leads to an overall mean improvement of $0.24 \mathrm{~mm}$, rising to $0.57 \mathrm{~mm}$ for fractures. The symmetric (in probability) $98 \%$ confidence interval of the mean improvement (derived by bootstrap resampling) is [0.143,0.385].

\section{Discussion}

The previously observed accuracy improvement 9] of around $0.4 \mathrm{~mm}$ between the statically ordered triplet approach and a global model disappears with a larger training set. A similar effect was noted in [2] comparing a standard and an hierarchical ASM. But the mean difference between the dynamic sub-model approach and a single global model is statistically significant (using a $98 \%$ bootstrap confidence interval on the mean difference). So there does appear to be a real improvement from the heuristic of first imposing the better fitting regions, and then using the constraints implied by them in subsequent (partial) searches. Further gains might possibly be derived from increasing the size of the candidate subset $N$, but this would of course increase the computation time of the algorithm.

With the new dynamic ordering method the mean location accuracy of 0.7 $\mathrm{mm}$ for normal (i.e. not fractured) vertebrae is comparable to typical manual precision. Over $95 \%$ of points in normal vertebrae are located to within $2 \mathrm{~mm}$. The performance on fractured vertebrae worsens, but remains quite respectable, and the mean of $1.23 \mathrm{~mm}$ is still comparable with manual precision on fractures. Fractured vertebrae present a more challenging problem, as the variation is much greater for pathological cases than for normals, and also when a vertebral endplate collapses a remnant of the stronger outer ring of cortical bone can provide a weaker outer edge to further confuse the search algorithm.

Nevertheless around $84 \%$ of points in fractured vertebrae are located to within $2 \mathrm{~mm}$ with the dynamic method. The remaining $16 \%$ in the tail of the distribution are due to a combination of under-training of the models, and false local minima. In the former case the problem is that the shape model cannot fit to some of the more extreme fractures. In the latter case, there appear to be problems with local minima where the top of a vertebra is erroneously fit to the bottom of the vertebra above (or vice versa). Fractured vertebrae may be prone to this failure mode, as the true edge of a collapsed vertebral body may well be further from the starting solution than the opposite side of its neighbour. It may ultimately prove necessary to search using multiple initialisations to segment pathological cases more reliably - for example by using a normal and a fractured candidate. However even here our framework could still be used, as multiple candidates of the same sub-model can essentially be treated in the same way as multiple different sub-models.

The accuracy is better than other comparable cited figures in the literature 8 8663. For example de Bruijne et al [3] obtained a mean point-to-contour accuracy of $1.4 \mathrm{~mm}$ on lumbar radiographs using shape particle filtering, whereas 
we achieve a mean accuracy of $0.8 \mathrm{~mm}$, even though DXA images are typically noisier than radiographs and have poorer resolution, and we also include the midthorax which has more soft-tissue clutter and a greater probability of fracture.

Our latest results confirm the feasibility of substantially automating vertebral morphometry measurements even with fractures or noisy images. However some work remains on extending the models and search process to cope with the more severe fractures. Furthermore the sub-model approach offers scope for greater gains on radiographic images, which have significant local variation in projective effects, which could be compensated for by varing the affine pose of the submodels.

\section{References}

1. Guermazi A, Mohr A, Grigorian M, Taouli B, and Genant HK. Identification of vertebral fractures in osteoporosis. Seminars in Musculoskeletal Radiology, 6(3):241$252,2002$.

2. Davatzikos $\mathrm{C}$ and Tao. Hierachical active shape models: Using the wavelet transform. IEEE Trans. Med. Imag., 22(3):414-423, 2003.

3. de Bruijne $M$ and Nielsen M. Image segmentation by shape particle filtering. In International Conference on Pattern Recognition, pages 722-725. IEEE Computer Society Press, 2004.

4. McCloskey E, Selby P, de Takats D, and et al. Effects of clodronate on vertebral fracture risk in osteoporosis: a 1-year interim analysis. Bone, 28(3):310-5, 2001.

5. R Eastell, SL Cedel, HW Wahner, BL Riggs, and LJ Melton. Classification of vertebral fractures. J Bone Miner Res, 6(3):207-215, 1991.

6. B. Howe, A. Gururajan, H. Sari-Sarraf, and R. Long. Hierarchical segmentation of cervical and lumbar vertebrae using a customized generalized hough transform and extensions to active appearance models. In Proc IEEE 6th SSIAI, pages 182-186, 2004.

7. Melton LJ III, Atkinson EJ, Cooper C, O'Fallon WM, and Riggs BL. Vertebral fractures predict subsequent fractures. Osteoporosis Int, 10:214-221, 1999.

8. Smyth PP, Taylor CJ, and Adams JE. Vertebral shape: automatic measurement with active shape models. Radiology, 211:571-578, 1999.

9. M.G. Roberts, T.F. Cootes, and J.E. Adams. Linking sequences of active appearance sub-models via constraints: an application in automated vertebral morphometry. In 14th British Machine Vision Conference, pages 349-358, 2003.

10. Cootes TF and Taylor CJ. Constrained active appearance models. In 8th International Conference on Computer Vision, volume 1, pages 748-754. IEEE Computer Society Press, July 2001.

11. Cootes TF, Edwards GJ, and Taylor CJ. Active appearance models. In Burkhardt $\mathrm{H}$ and Neumann B, editors, 5th European Conference on Computer Vision, volume 2, pages 484-498. Springer (Berlin), 1998. 